\title{
MicroRNA-377 predicts poor clinical outcome of gastric cancer and induces tumorigenesis by targeting multiple tumor-suppressor genes
}

\author{
XU WEN ${ }^{1}$, JIAN-QIU WU ${ }^{2}$, WEI PENG ${ }^{2}$, JI-FENG FENG ${ }^{2}$ and JIN-HAI TANG ${ }^{1}$ \\ Departments of ${ }^{1}$ General Surgery and ${ }^{2}$ Medical Oncology, Nanjing Medical University \\ Affiliated Cancer Hospital, Cancer Institute of Jiangsu Province, Nanjing 210009, P.R. China
}

Received March 13, 2015; Accepted April 27, 2015

DOI: $10.3892 /$ or.2015.3981

\begin{abstract}
Gastric cancer (GC) is a major cause of cancer mortality worldwide. MicroRNAs are evolutionally conserved small non-coding RNAs that are critical for the regulation of gene expression. The aberrant expression of microRNA (miRNA) is involved in tumorigenesis and prognosis. In the present study, the clinical significance of miR-377 was assessed using RT-qPCR and MTT assay. The results showed that the expression of miR-377 was upregulated in GC compared with normal gastric tissues, and its expression level was increased in GC cell lines compared with normal gastric cells. In addition, there was a significant association between miR-377 expression and clinicopathological characteristics, in particular distant metastasis, TNM stage and early recurrence. GC patients with a higher miR-377 expression showed significantly poorer overall survival (OR) and shorter time to recurrence than those with a lower miR-377 expression. The Cox regression analysis identified miR-377 overexpression as an independent prognostic factor for GC. Overexpression of miR-377 in MKN-45 GC cells significantly promoted cell proliferation, whereas the suppression of miR-377 inhibited these effects. Furthermore, miR-377 downregulated p53, PTEN and TIMP1 expression by directly targeting the 3'-untranslated region of these target genes. Collectively, miR-377 potentially served as a new molecular predictive biomarker of GC tumorigenesis and prognosis, which may be useful in targeted therapy and the prognosis of GC patients.
\end{abstract}

\section{Introduction}

Gastric cancer (GC) remains the fourth most commonly diagnosed cancer and is the second leading cause of

Correspondence to: Dr Jin-Hai Tang, Department of General Surgery, Nanjing Medical University Affiliated Cancer Hospital, Cancer Institute of Jiangsu Province, Nanjing 210009, P.R. China E-mail: willgeek01@163.com

Key words: gastric cancer, microRNA-377, tumorigenesis, prognosis, biomarker cancer-associated mortality worldwide (1). The carcinogenesis of GC is complicated and involves the dysregulation of oncogenes and tumor suppressors (2). Many molecules are responsible for GC tumorigenesis, and despite advances in surgical and chemotherapeutic interventions, the overall 5-year survival for GC patients remains low (30\%) and the recurrence rate high (3-5). Thus, identifying new biomarkers or factors that improve the diagnosis of GC prior to tumorigenesis and predict prognosis responsible for cancer recurrence in patients with GC is crucial.

A number of microRNA (miRNA) genes have been identified in the human genome and it has been suggested that at least $50 \%$ of all protein-encoding genes are regulated by miRNA (6). Mature miRNAs are 21-22 nucleotides in size and affect the post-translational expression of genes by interacting with complementary target sites within the 3'-untranslated region of the messenger RNA (7). Recent findings have shown the role of miRNAs in a variety of basic biological and pathological processes (8) and the association of miRNA signatures with human diseases has been established (9). miR-377 can have a critical role in the pathophysiology of diabetic nephropathy (10) and human nucleus pulposus cells (11). miR-377 was responsible for metabolizing the excess heme generated during hemolysis (12). miR-377 may be required for glioblastoma multiforme (GBM) development and serve as a therapeutic target for the treatment of GBM (13). However, the expression of miR-377 in GC and its prognostic values remain to be elucidated.

In the present study, we evaluated the clinical significance of miR-377. The expression level of miR-377 was overexpressed in GC tissues and cell lines. Furthermore, the correlation between the expression level of miR-377 and clinicopathological characteristics was analyzed. The prognostic value of miR-377 on the prognosis of GC patients was also estimated. Further analyses showed that the overexpression of miR-377 promoted GC cell proliferation. Moreover, p53, PTEN and TIMP1 were identified as direct targets of miR-377.

\section{Materials and methods}

Patients and specimens. This study was approved by the Medical Ethics Committee of Jiangsu Cancer Hospital 
(Nanjing, China) and written informed consent was obtained from all of the patients. The specimens were handled and made anonymous according to the ethical and legal standards. Paired tissue specimens (tumor and adjacent normal mucosa) from 102 patients with GC were obtained and histologically confirmed by a pathologist at Jiangsu Cancer Hospital and the First People's Hospital of Yunnan Province from January, 2007 to December, 2010. The samples were derived from patients who had not received adjuvant treatment including radiotherapy or chemotherapy prior to surgery in order to eliminate potential treatment-induced changes to gene expression profiles. After excision, the tissue specimens were immediately frozen in liquid nitrogen for subsequent analysis. The specimens were stained with hematoxylin and eosin and examined histopathologically. The sections that consisted of $>80 \%$ carcinoma cells were used to prepare the total RNA. Clinicopathological information, including gender, age, tumor size, depth of invasion, lymph node invasion, distant metastasis, TNM stage, tumor differentiation and early recurrence was available for all the patients.

Cell culture. The human HGC-27, MKN-45, BGC-823, AGS, SGC-7901, MGC80-3, NCI-N87 and SNU-1 gastric cancer cell lines, and GES-1 normal gastric cell line were obtained from the American Type Culture Collection (ATCC; Manassas, VA, USA). MKN-45, BGC-823, AGS, SGC-7901 and SNU-1 cells were grown in Dulbecco's modified Eagle's medium (DMEM) and HGC-27, MGC80-3, NCI-N87 and GES-1 were grown in RPMI-1640. In each case, the medium was supplemented with $15 \%$ fetal calf serum (FCS), $100 \mathrm{U} / \mathrm{ml}$ penicillin and $100 \mathrm{U} / \mathrm{ml}$ streptomycin. The cells were incubated at $37^{\circ} \mathrm{C}$ in a humidified incubator containing 5\% $\mathrm{CO}_{2}$.

Reverse transcriptase-quantitative PCR (RT-qPCR). Approximately $40 \mathrm{mg}$ of tissue sample or harvested cells $\left(2 \times 10^{6}\right)$ was homogenized in $1 \mathrm{ml}$ TRIzol reagent (Invitrogen, Carlsbad, CA, USA), according to the manufacturer's instructions. The purity and concentration of RNA were determined using a NanoDrop 1000 spectrophotometer (Thermo Fisher Scientific, Wilmington, DE, USA). RNA ( $2 \mu \mathrm{g})$ was reverse transcribed from each sample to produce cDNA using the PrimeScript RT reagent kit (Takara Biotechnology, Dalian, China). Quantitative PCR was employed to determine the relative expression level of target genes using the SYBR Premix ExTaq II kit (Takara) on the 7300 Real-Time PCR systems (Applied Biosystems, Carlsbad, CA, USA). The PCR cycling profile was denatured at $95^{\circ} \mathrm{C}$ for $30 \mathrm{sec}$, followed by 40 cycles of annealing at $95^{\circ} \mathrm{C}$ for $5 \mathrm{sec}$ and extension at $60^{\circ} \mathrm{C}$ for $34 \mathrm{sec}$. Small nucleolar RNA U6 was used as an internal standard for normalization. The cycle threshold (CT) value was calculated. The $2^{-\Delta \mathrm{CT}}\left(\Delta \mathrm{CT}=\mathrm{CT}_{\text {miR-377 }}-\mathrm{CT}_{\mathrm{U} 6 \mathrm{RNA}}\right)$ method was used to quantify the relative amount of miR-377.

miRNA mimics, plasmids and transfection. The miR-377 mimic, miR-377 mutant (miR-377-mut) and miR-377-inhibitor (miR-377-in) were purchased from the Shanghai GenePharma, Co. (Shanghai, China), together with the negative control (miR-control). The transfection of miRNAs $(50 \mathrm{nM})$ was performed using X-tremeGENE (Roche, Reinach, Switzerland) according to the manufacturer's instructions. The 3'-UTR of p53, PTEN and TIMP1 was PCR-amplified from MKN-45 genomic DNA and cloned downstream of the luciferase gene in the pGL vector (Promega, Madison, WI, USA). For the reporter assay, the cells were cultured in 96-well plates and transfected with luciferase reporters $(50 \mathrm{ng})$ and $50 \mathrm{nM}$ of miR-control, miR-377 mimics or miR-377-mut. After $48 \mathrm{~h}$, luciferase activity was measured using the dual-luciferase reporter system (Promega). The Renilla activity was used as an internal control. Each transfection was performed in triplicate.

MTT assay. The cells were seeded in 96-well plates (2x10 $3 /$ well) $24 \mathrm{~h}$ after transfection. MTT (Beyotime, Haimen, China) was added to each well and the cells were cultured for $4 \mathrm{~h}$ at $37^{\circ} \mathrm{C}$. The reaction was stopped by $150 \mu \mathrm{l}$ DMSO and the optical density at $490 \mathrm{~nm}$ was detected on a microplate reader (Thermo Fisher Scientific, Kalamazoo, MI, USA).

Colony formation assay. Five hundred of each transfected cells were plated in 6-well plates and cultured for 14 days without any disturbance. The cells were stained with $0.5 \%$ crystal violet for $1 \mathrm{~h}$ at $37^{\circ} \mathrm{C}$. Visible colonies were counted in four different fields and the mean value was calculated.

Cell cycle analysis. The cells were seeded into 6-well plates with a density of $1 \times 10^{5}$ cells/well after transfection and maintained in DMEM/RPMI-1640 containing 15\% FCS. Cultured cells were trypsinized after $48 \mathrm{~h}$ and fixed with $70 \%$ ethanol at $4^{\circ} \mathrm{C}$ overnight before being stained with propidium iodide (PI) (Invitrogen). DNA contents were detected using an LSRII flow cytometer (BD Biosciences, San Jose, CA, USA). Data were analyzed by Flow Jo (Tree Star, Ashland, OR, USA). TargetScan, miRanda and DIANA were used to determine the putative human protein-coding gene targets of miR-377.

Luciferase reporter assays. MKN-45 cells were co-transfected with miR-377 or control or the mutated 3'-UTR (Mut) of p53, PTEN and TIMP1. After $48 \mathrm{~h}$, the cells were collected and luciferase activity was assayed using the dual-luciferase assay system (Promega).

Protein isolation and western blot analysis. Total proteins were extracted with RIPA lysis buffer with proteinase/phosphatase inhibitors (Beyotime). The lysate was separated by $10 \%$ sodium dodecyl sulfate polyacrylamide gel electrophoresis and the gel was blotted onto a PVDF membrane (Bio-Rad, Hercules, CA, USA). The membrane was blocked in $1 \%$ BSA and then incubated with one of the following antibodies: antip53 (Cellsignal, no. 2527), anti-PTEN (Cellsignal, no. 5384), anti-TIMP1 (Cellsignal, no. 8946) or anti-GAPDH (Cellsignal, no. 2118). Goat radish peroxidase-conjugated anti-rabbit IgG (Cellsignal, no. 2975) were incubated as the secondary antibodies. Subsequent visualization was detected using a SuperSignal West Femto Maximum Sensitivity Substrate (Thermo Fisher Scientific).

Statistical analysis. Data are presented as means \pm SD. A comparison of the level of miR-377 expression between GC and adjacent normal tissue was performed using the Wilcoxon test. The correlation between the expression of miR-377 and clinicopathological characteristics was assessed using a 

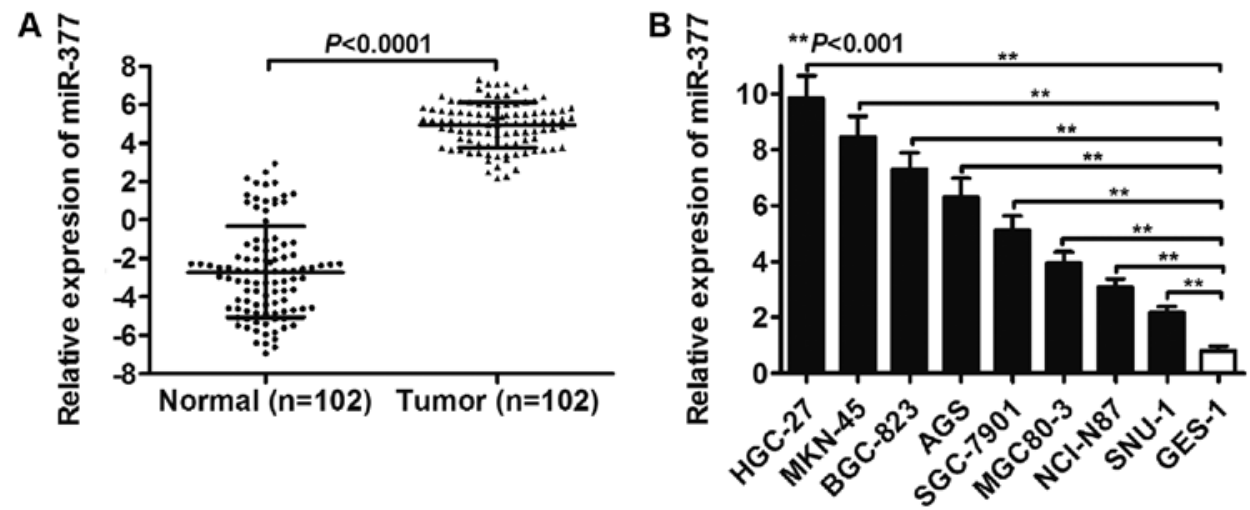

Figure 1. Expression of miR-377 in GC tissues and cell lines. (A) Significant increase in the miR-377 level was found in GC tumors as compared to adjacent normal tissues ( $\mathrm{n}=102$, P<0.0001). (B) Levels of miR-377 in HGC-27, MKN-45, BGC-823, AGS, SGC-7901, MGC80-3, NCI-N87 and SNU-1 GC cell lines are higher than the levels of miR-377 in the human GES-1 normal cells $(\mathrm{P}<0.001)$. Mean \pm SD represents three independent experiments.

two-sample Student's t-test. The postoperative survival rate was analyzed using the Kaplan-Meier method and differences in survival rates were assessed with the log-rank test. A Cox proportional hazards analysis was performed to calculate the hazard ratio (HR) and the $95 \%$ confidence interval (CI) to determine the association between miR-377 expression and survival. In addition, a multivariate Cox regression was performed to adjust for other covariates. The tests were two-tailed and results with $\mathrm{P}<0.05$ were considered statistically significant. Statistical analyses were performed using SPSS 19.0 software (SPSS, Chicago, IL, USA).

\section{Results}

miR-377 was overexpressed in human GC tissues and cell lines. As shown in Fig. 1A, the overall expression level of miR-377 was determined by RT-qPCR in 102 paired normal and GC tissues. A significantly different expression level of miR-377 between the normal and tumor groups was identified $(\mathrm{P}<0.0001)$. Furthermore, miR-377 expression in tumor tissues showed elevated levels of miR-377 compared to the corresponding normal tissues, with an average increase of 9.62-fold. Levels of miR-377 in the BGC-823, AGS, SGC-7901, MGC80-3, NCI-N87 and SNU-1 GC cell lines HGC-27, MKN-45, were also higher than that in the normal GES-1 cell line $(\mathrm{P}<0.001$, Fig. 1B).

Correlations between miR-377 expression and clinicopathological characteristics. To identify the clinical relevance of miR-377 expression in GC, the correlations between miR-377 expression and clinicopathological parameters such as gender, age, tumor size, depth of invasion, lymph node invasion, distant metastasis, TNM stage, tumor differentiation and early recurrence were examined (Table I). Of the 102 GC patients, 82 cases were included in the miR-377-high group and the remaining 20 cases were included in the miR-377-low group. As shown in Table I, the results demonstrated that a high expression of miR-377 was significantly correlated with distant metastasis $(\mathrm{P}=0.034)$, TNM stage $(\mathrm{P}=0.030)$ and early recurrence $(\mathrm{P}=0.044)$. However, there was no significant association between miR-377 expression and other clinicopathological chacteristics, such as gender, age and tumor size, depth of invasion, lymph node invasion and tumor differentiation $(\mathrm{P}>0.05)$.

Prognostic value of $\mathrm{miR}$-377. The results showed that miR-377 was a prognostic factor for overall survival (OS) and time to recurrence (TTR) in GC. The Log-rank test results showed that the 3- and 5-year OS rates in the group with miR-377 upregulation were significantly lower than those in the group with miR-377 downregulation $(\mathrm{P}=0.0106)$ (Fig. 2A). Similarly, the cumulative TTR rates in the miR-377 upregulation group were significantly higher than those in the miR-377 downregulation group ( $\mathrm{P}=0.0004)$ (Fig. 2B). Furthermore, the Cox regression analysis suggested that distant metastasis and miR-377 expression status were independent factors that affected $\mathrm{OS}(\mathrm{P}=0.027, \mathrm{HR}=1.62,95 \% \mathrm{CI}=1.13-2.78)$, while miR-377 expression status was an independent factor for TTR $(\mathrm{P}=0.022, \mathrm{HR}=2.14,95 \% \mathrm{CI}=0.87-4.42)$ (Table II). Collectively, these data suggested that miR-377 was an independent prognostic biomarker in GC.

miR-377 promotes $G C$ cell proliferation. To examine the role of miR-377 in GC tumorigenesis, we examined the effect of miR-377 overexpression and inhibition on the proliferation of MKN-45 GC cell lines. The cells were transfected with miR-377 mimic (miR-377), miR-377 inhibitor (miR-377-in) or the miR scramble control oligonucleotides (Control). RT-qPCR showed that miR-377 was significantly increased in the cells transfected with miR-377 mimics and decreased in the miR-377 inhibitor group compared with the control (Fig. 3A). The MTT assay showed that the overexpression of miR-377 significantly promoted the proliferation of MKN-45 cells, whereas the inhibition of miR-377 suppressed cell proliferation (Fig. 3B). The colony formation assay was performed to further confirm the effect of miR-377 on GC cell proliferation, and data indicated that the overexpression of miR-377 significantly increased colony numbers in MKN-45 cell cultures, whereas the knockdown of miR-377 expression obviously decreased colony forma- 
Table I. Clinicopathological parameters of GC patients and the correlation with miR-377 expression.

\begin{tabular}{|c|c|c|c|c|}
\hline \multirow[b]{2}{*}{ Characteristics } & \multirow[b]{2}{*}{ No. } & \multicolumn{2}{|c|}{ miR-377 expression } & \multirow[b]{2}{*}{ P-value } \\
\hline & & Low & High & \\
\hline Gender & & & & 0.328 \\
\hline Male & 67 & 15 & 52 & \\
\hline Female & 35 & 5 & 30 & \\
\hline Age (years) & & & & 0.936 \\
\hline$<60$ & 62 & 12 & 50 & \\
\hline$\geq 60$ & 40 & 8 & 32 & \\
\hline Tumor size $(\mathrm{cm})$ & & & & 0.174 \\
\hline$<4.5$ & 63 & 15 & 48 & \\
\hline$\geq 4.5$ & 39 & 5 & 34 & \\
\hline Depth of invasion & & & & 0.942 \\
\hline $\mathrm{T} 1, \mathrm{~T} 2$ & 21 & 4 & 17 & \\
\hline $\mathrm{T} 3, \mathrm{~T} 4$ & 81 & 16 & 65 & \\
\hline Lymph node invasion & & & & 0.085 \\
\hline Absent & 78 & 18 & 60 & \\
\hline Present & 24 & 2 & 22 & \\
\hline Distant metastasis & & & & 0.034 \\
\hline Absent & 40 & 12 & 28 & \\
\hline Present & 62 & 8 & 54 & \\
\hline TNM stage & & & & $\mathbf{0 . 0 3 0}$ \\
\hline $\mathrm{I}, \mathrm{II}$ & 35 & 11 & 24 & \\
\hline III,IV & 67 & 9 & 58 & \\
\hline Tumor differentiation & & & & 0.258 \\
\hline Well, moderate & 60 & 14 & 46 & \\
\hline Poor, mucinous & 42 & 6 & 36 & \\
\hline Early recurrence & & & & 0.044 \\
\hline No & 41 & 12 & 29 & \\
\hline Yes & 61 & 8 & 53 & \\
\hline
\end{tabular}

Bold, statistically significant $(\mathrm{P}<0.05)$.

A

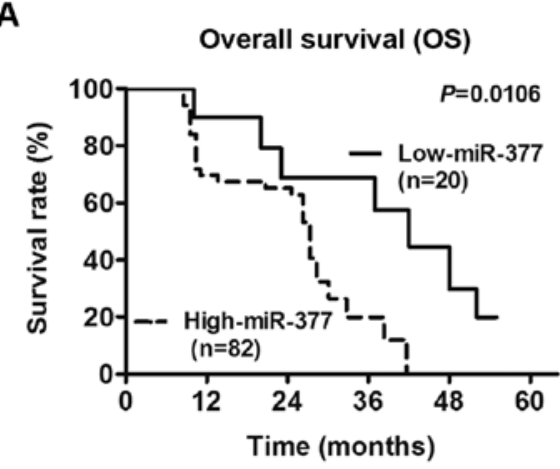

B

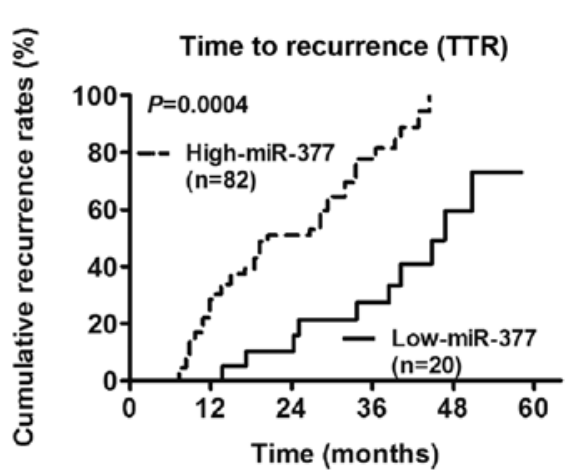

Figure 2. Overall survival (OS) and time to recurrence (TTR) of patients with GC based on miR-377 expression status. Kaplan-Meier survival curves for (A) OS (P=0.0106) and (B) TTR (P=0.0004).

tion (Fig. 3C). Furthermore, we assessed the cell cycle by flow cytometry. As shown in Fig. 3D, the transfection of miR-377 decreased the percentage of cells in G1 peak but increased that in the S peak. Similarly, miR-377-in led to cell cycle arrests in MKN-45 cells. Collectively, these results showed that miR-377 promotes GC cell growth. 
Table II. Univariate and multivariate analyses of prognostic variables of overall survival and time to recurrence in GC patients.

\begin{tabular}{|c|c|c|c|c|c|c|}
\hline \multirow[b]{2}{*}{ Variables } & \multicolumn{3}{|c|}{ Univariate analysis } & \multicolumn{3}{|c|}{ Multivariate analysis } \\
\hline & HR & $95 \% \mathrm{CI}$ & P-value & HR & $95 \% \mathrm{CI}$ & P-value \\
\hline Overall survival & 3.56 & $3.28-4.34$ & 0.713 & & & \\
\hline Gender (male vs. female) & 2.77 & $2.65-3.98$ & 0.248 & & & \\
\hline Age $(<60$ vs. $\geq 60)$ & 2.81 & $2.36-3.51$ & 0.057 & & & \\
\hline Tumor size $(<4.5$ vs. $\geq 4.5)$ & 2.15 & $1.76-3.01$ & 0.323 & & & \\
\hline Depth of invasion (T1,T2 vs. T3,T4) & 1.88 & $1.45-3.54$ & 0.082 & & & \\
\hline Lymph node invasion (absent vs. present) & 2.55 & $1.76-4.22$ & 0.013 & 1.73 & $1.26-2.63$ & 0.091 \\
\hline Distant metastasis (absent vs. present) & 1.39 & $0.88-3.19$ & 0.025 & 1.82 & $1.04-3.15$ & 0.016 \\
\hline TNM stage (I,II vs. III,IV) & 1.14 & $0.63-1.79$ & 0.014 & 0.96 & $0.65-1.61$ & 0.065 \\
\hline $\begin{array}{l}\text { Tumor differentiation } \\
\text { (Well, moderate vs. poor, mucinous) }\end{array}$ & 1.37 & $1.01-2.63$ & 0.419 & & & \\
\hline Early recurrence (no vs. yes) & 1.19 & $0.88-2.69$ & 0.035 & 1.65 & $1.17-3.05$ & 0.043 \\
\hline miR-377 expression (low vs. high) & 1.48 & $1.09-2.43$ & 0.006 & 1.62 & $1.13-2.78$ & $\mathbf{0 . 0 2 7}$ \\
\hline \multicolumn{7}{|l|}{ Time to recurrence } \\
\hline Gender (male vs. female) & 2.08 & $1.86-2.83$ & 0.216 & & & \\
\hline Age $(<60$ vs. $\geq 60)$ & 2.68 & $2.38-3.59$ & 0.971 & & & \\
\hline Tumor size $(<4.5$ vs. $\geq 4.5)$ & 4.02 & $3.21-4.46$ & 0.317 & & & \\
\hline Depth of invasion (T1,T2 vs. T3,T4) & 2.94 & $2.62-3.94$ & 0.462 & & & \\
\hline Lymph node invasion (absent vs. present) & 3.27 & $2.95-4.09$ & 0.013 & 2.97 & $1.89-3.32$ & 0.093 \\
\hline Distant metastasis (absent vs. present) & 2.38 & $0.52-4.25$ & 0.045 & 3.71 & $3.39-4.67$ & 0.232 \\
\hline TNM stage (I,II vs. III,IV) & 1.92 & $0.67-4.29$ & 0.018 & 2.51 & $2.19-3.33$ & 0.307 \\
\hline $\begin{array}{l}\text { Tumor differentiation } \\
\text { (Well, moderate vs. poor, mucinous) }\end{array}$ & 1.56 & $0.18-3.08$ & 0.054 & & & \\
\hline Early recurrence (no vs. yes) & 1.23 & $0.54-2.61$ & 0.027 & 2.03 & $0.17-3.85$ & 0.041 \\
\hline miR-377 expression (low vs. high) & 0.77 & $0.41-2.87$ & 0.012 & 2.14 & $0.87-4.42$ & 0.022 \\
\hline
\end{tabular}

HR, hazard ratio; 95\% CI, 95\% confidence interval. Bold, statistically significant $(\mathrm{P}<0.05)$.

miR-377 directly targets p53, PTEN and TIMP1. To gain insight into the biological implications of miR-377 on GC tumorigenesis, we used TargetScan, miRanda and DIANA for the putative human protein-coding gene targets of miR-377. The tumor-suppressor genes p53, PTEN and TIMP1 were predicted to have miR-377-binding elements in their 3'-UTRs with high fidelity scores (Fig. 4A). To assess whether the predicted miR-377-binding sites in the 3'-UTR of the three target genes were responsible for miR-377 regulation, we cloned the 3'-UTR regions downstream of a luciferase reporter gene and co-transfected these vectors together with control, miR-377 and miR-377-mut into MKN-45 cells, respectively. The luciferase activity of cells transfected with miR-377 was significantly decreased compared with the control. However, the mutation of miR-377 clearly abrogated the repression of the luciferase activity (Fig. 4B). To investigate whether miR-377 regulated these targets, the protein expression levels of p53, PTEN and TIMP1 were assessed by western blot analysis. As shown in Fig. 4C, the overexpression of miR-377 markedly altered the protein levels of p53, PTEN and TIMP1, whereas the protein expression of these target proteins was not affected by mutant miR-377. These results suggested that p53, PTEN and TIMP1 are direct targets of miR-377 and these three targets may mediate the promotive effect of miR-377 on tumorigenesis.

\section{Discussion}

$\mathrm{GC}$ is one of the most common and lethal cancers, with a high relapse rate $(1,14)$. Therefore, it is essential to develop novel, prognostic factors and therapeutic strategies. The outcome of GC patients is determined primarily by the presence or absence of metastasis (15). Thus, identification of the precise molecular mechanisms that modulate malignant transformation is necessary. Identification of miRNA molecular profiles associated with the prognosis of patients with GC only shed light on the elucidation of the underlying biological mechanisms involved in the development or progression of the disease, and provide the opportunity to identify novel targets for GC diagnosis and treatment (16). To the best of our knowledge, we have shown for the first time that miR-377 was frequently upregulated in GC tissues than in their normal adjacent tissues. Furthermore, the results suggest that miR-377 is involved in the progression of GC. Of note, we found that a high expression of miR-377 was a significant predictor of OS and TTR. 
A

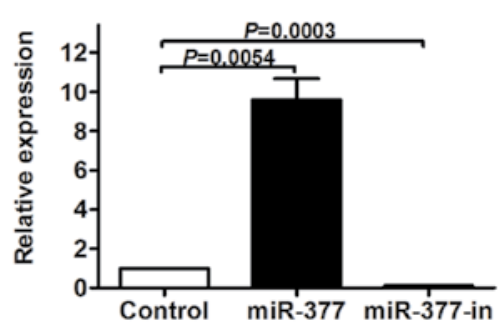

C

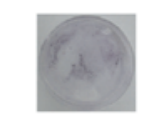

B

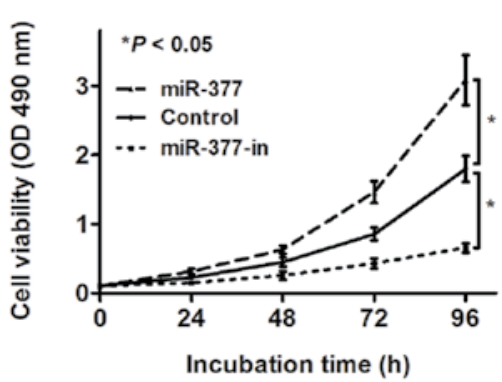

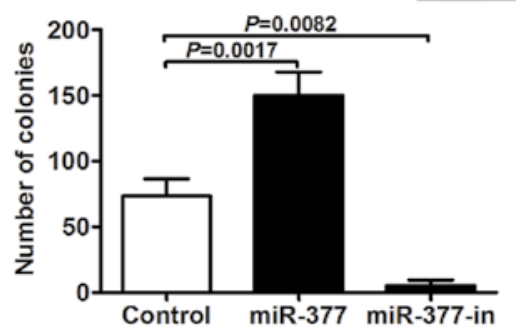

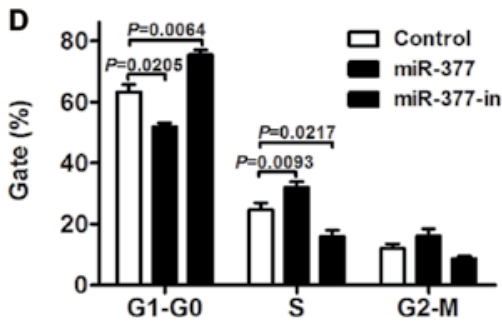

Figure 3. MiR-377 promotes cell growth in MKN-45 cells. (A) RT-qPCR analyses of miR-377 levels in control-, miR-377- and miR-377-in-transfected MKN-45 cells. (B) Effects of miR-377 and miR-377-in on tumor cell proliferation at 0, 24, 48, 72 and $96 \mathrm{~h}$ using the MTT assay. (C) Representative quantification of colony formation in MKN-45 cells transfected with control, miR-377 and miR-377-in. (D) Cell cycle profiles of MKN-45 cells transfected with control, miR-377 and miR-377-in. Data are shown as mean \pm SD of three independent experiments.

A

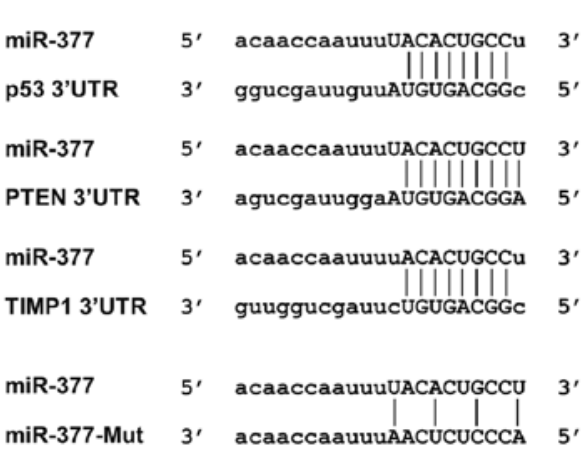

C

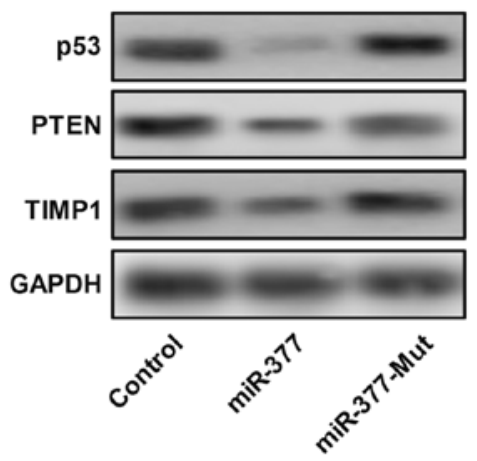

B
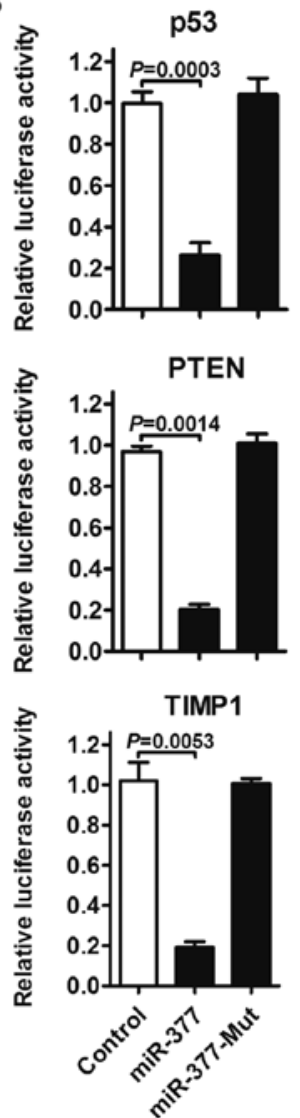

Figure 4. p53, PTEN and TIMP1 are direct targets of miR-377. (A) Sequences of the putative miR-377 binding sites in the 3'-UTR of p53, PTEN and TIMP1. Ten nucleotides of miR-377-mut were mutated to prevent the broad binding of miR-377 and its targets. (B) Luciferase assay of MKN-45 cells transfected with hRluc-p53-3'UTR, hRluc-PTEN-3'UTR or hRluc-TIMP1-3'UTR and $50 \mathrm{nM}$ of control, miR-377 or miR-377-mut, respectively. (C) Western blot analysis of p53, PTEN and TIMP1 in MKN-45 cells transfected with $50 \mathrm{nM}$ control, miR-377 or the miR-377-mut. Data are shown as mean \pm SD of three independent experiments. MicroRNA-377 predicts poor clinical outcome of gastric cancer and induces tumorigenesis by targeting multiple tumor-suppressor genes. 
Previous findings suggested that miR-377 plays a vital role in stress responses during pathological conditions, such as diabetes and aging $(9,10,17)$. Overexpression of miR-377 has been observed in human mesangial cells, and was associated with an increased expression of the matrix protein, fibronectin, which is accumulated in excess in diabetic nephropathy (10). In the present study, we first confirmed that miR-377 was frequently upregulated in GC tissues than in their normal adjacent mucosa, and identified a high miR-377 expression as a valid factor associated with advanced tumor stages. These data suggest the potential of miR-377 to serve as a molecular target for GC therapy, especially for tumors with a high potency of metastasis.

The correlation between the miR-377 expression level and clinicopathological values of GC remains unclear. In the present study, we first investigated the relationship of miR-377 expression with clinicopathological characteristics and prognosis. The miR-377 expression was found to be associated with distant metastasis, TNM stage and early TTR, suggesting that miR-377 was involved in the tumorigenesis, development, progression and metastasis of GC. Of note, miR-377 expression was significantly associated with the OS and TTR of GC patients. In support of this, the Kaplan-Meier analysis of overall survival showed that patients whose tumors with a higher miR-377 expression had a significantly worse OS and TTR, indicating that a high miR-377 level is a biomarker of poor prognosis for GC patients. Moreover, the Cox proportional hazards model showed that miR-377 was an independent marker of poor OS and TTR of the known clinical prognostic indicators including distant metastasis, TNM stage and early TTR. Therefore, constitutes a molecular prognostic marker for GC patients, identifying higher risk of death in patients. Thus, good candidates are to receive a more aggressive treatment. To the best of our knowledge, this is the first report to describe the clinical significance of miR-377 in TTR and prognosis of GC patients.

To examine the significance of miR-377 upregulation in GC, several assays were performed. First, we conducted a cell growth assay on MKN-45 cells to assess whether the differential expression of miR-377 affected proliferation. We found that the upregulation of miR-377 expression significantly promoted proliferation and that inhibition of miR-377 suppressed cell proliferation. When assessing the cell cycle of MKN-45 cells by flow cytometry, it was found that miR377-mimic-transfected cells yielded a population with more cells in the S-phase, and fewer cells in G1-phase, and that inhibition of miR-377 led to cell cycle arrest. The S-phase is the period in which a cell performs DNA replication and the G1/S transition is a major checkpoint in the regulation of the cell cycle (18). Our data suggest that miR-377 affects mechanisms that control the G1/S transition in GC cells.

To investigate the downstream effects of miR-377 overexpression in GC cells, we developed a luciferase-reporter system to assess whether miR-377 can regulate known tumor suppressors and found that miR-377 targets p53, PTEN and TIMP1. p53 is an important tumor-suppressor gene and responds to diverse cellular stresses to regulate the expression of target genes, especially inducing cell cycle arrest and apoptosis (19). Mutations in p53 are associated with a variety of human cancers (20). The p53 has been previously reported to be downregulated in $\mathrm{GC}$ tissues, and restoring its expression inhibits growth and reduces clonogenic activity in $\mathrm{GC}$ cell lines (21). The result is consistent with our observations that MKN-45 cells have a downregulated p53 expression caused by miR-377, and increased proliferation ability. PTEN has been identified as a tumor suppressor that is mutated in a large number of cancers at high frequency (22). The protein encoding this gene is phosphatidylinositol-3,4,5-trisphosphate 3 -phosphatase (23). It negatively regulates the intracellular levels of phosphatidylinositol-3,4,5-trisphosphate in cells and negatively regulates the AKT/PKB signaling pathway (24). Thus, it seems likely that our observation of increased cell numbers in the S-phase and decreased numbers in the G1-phase was caused by the modulation of PTEN by miR-377. TIMP1 has been demonstrated to be a suppressor of tumorigenesis and progression (25). It is a natural inhibitor of the matrix metalloproteinases (MMPs), which are able to promote cell proliferation and may also have an antiapoptotic function (26). Dysregulation of TIMP1 has been found in several types of cancer (27). In the present study, TIMP1 was found to be downregulated by miR-377 and when miR-377-overexpressing cells exhibited increased growth activity. This result suggests that the dysregulation of TIMP1 by miR-377 is a mechanism associated with this process. The present study provides confounding evidence that an upregulated expression of miR-377 in GC modulates core mechanisms in the control of cell cycle progression and proliferation, by inhibiting the expression of tumor suppressor p53, PTEN and TIMP1. The differential expression of miR-377 in GC can potentially be utilized in diagnostic applications and therapeutic interventions.

In conclusion, to the best of our knowledge, the results of the present study have, for the first time, demonstrated that miR-377 was overexpressed in GC tissue and cell lines and associated with tumorigenesis and poor prognosis. The present study also demonstrated that miR-377 was an independent prognostic factor of patients with GC. Of note, the upregulated expression of miR-377 in GC modulates core mechanisms in the control of proliferation and cell cycle progression, by inhibiting expression of tumor suppressor p53, PTEN and TIMP1. The differential expression of miR-377 in GC can be a candidate therapeutic target and a potential biomarker for the diagnosis and prognosis in GC.

\section{Acknowledgements}

The present study was supported by grants from the Agency of Jiangsu Province Science and Technology (no. 2013035) and the Research Office of Jiangsu Cancer Hospital (no. ZK201401).

\section{References}

1. Gibson CJ, Britton KA, Miller AL and Loscalzo J: Clinical problem-solving. Out of the blue. N Engl J Med 370: 1742-1748, 2014.

2. Wu WKK, Lee CW, Cho CH, Fan D, Wu K, Yu J and Sung JJ: MicroRNA dysregulation in gastric cancer: A new player enters the game. Oncogene 29: 5761-5771, 2010.

3. Song JH and Meltzer SJ: MicroRNAs in pathogenesis, diagnosis, and treatment of gastroesophageal cancers. Gastroenterology 143: $35-47,2012$ 
4. Lin X, Yang M, Xia T and Guo J: Increased expression of long noncoding RNA ABHD11-AS1 in gastric cancer and its clinical significance. Med Oncol 31: 42, 2014.

5. Shen L, Shan YS, Hu HM, Price TJ, Sirohi B, Yeh KH, Yang YH, Sano T, Yang HK, Zhang X, et al: Management of gastric cancer in Asia: Resource-stratified guidelines. Lancet Oncol 14: e535-e547, 2013.

6. Chitwood DH and Timmermans MC: Small RNAs are on the move. Nature 467: 415-419, 2010.

7. Morris KV, Chan SW, Jacobsen SE and Looney DJ: Small interfering RNA-induced transcriptional gene silencing in human cells. Science 305: 1289-1292, 2004

8. Cai H, Yuan Y, Hao YF, Guo TK, Wei X and Zhang YM: Plasma microRNAs serve as novel potential biomarkers for early detection of gastric cancer. Med Oncol 30: 452, 2013.

9. Yu X, Luo L, Wu Y, Yu X, Liu Y, Yu X, Zhao X, Zhang X, Cui L, Ye G, et al: Gastric juice miR-129 as a potential biomarker for screening gastric cancer. Med Oncol 30: 365, 2013.

10. Wang Q, Wang Y, Minto AW, Wang J, Shi Q, Li X and Quigg RJ MicroRNA-377 is up-regulated and can lead to increased fibronectin production in diabetic nephropathy. FASEB J 22: 4126-4135, 2008.

11. Wen ZL, Huang W, Feng YL, Wang Y, Liang J, Cai W, Kang K, Chang D, Zhu P, Milliard RW, et al: MicroRNA-377 regulates angiogenesis by targeting VEGF: Implications for mesenchymal stem cells based therapy in ischemic heart disease. Circulation: $128,2013$.

12. Li N, Liu Q, Su Q, Wei C, Lan B, Wang J, Bao G, Yan F, Yu Y, Peng B, et al: Effects of legumain as a potential prognostic factor on gastric cancers. Med Oncol 30: 621, 2013.

13. Orditura M, Galizia G, Sforza V, Gambardella V, Fabozzi A, Laterza MM, Andreozzi F, Ventriglia J, Savastano B, Mabilia A, et al: Treatment of gastric cancer. World J Gastroenterol 20: 1635-1649, 2014

14. Vicentini C, Fassan M, D'Angelo E, Corbo V, Silvestris N, Nuovo GJ and Scarpa A: Clinical application of microRNA testing in neuroendocrine tumors of the gastrointestinal tract. Molecules 19: 2458-2468, 2014.

15. Beckman JD, Chen C, Nguyen J, Thayanithy V, Subramanian S, Steer CJ and Vercellotti GM: Regulation of heme oxygenase-1 protein expression by miR-377 in combination with miR-217. J Biol Chem 286: 3194-3202, 2011.

16. Zhang R, Luo H, Wang S, Chen W, Chen Z, Wang HW, Chen Y, Yang J, Zhang X, Wu W, et al: MicroRNA-377 inhibited prolife ration and invasion of human glioblastoma cells by directly targeting specificity protein 1. Neuro Oncol 16: 1510-1522, 2014
17. Tsirimonaki E, Fedonidis C, Pneumaticos SG, Tragas AA, Michalopoulos I and Mangoura D: PKCE signalling activates ERK1/2, and regulates aggrecan, ADAMTS5, and miR377 gene expression in human nucleus pulposus cells. PLoS One 8: e82045, 2013.

18. Bertoli C, Skotheim JM and de Bruin RAM: Control of cell cycle transcription during G1 and S phases. Nat Rev Mol Cell Biol 14: 518-528, 2013.

19. Duffy MJ, Synnott NC, McGowan PM, Crown J, O'Connor D and Gallagher WM: p53 as a target for the treatment of cancer. Cancer Treat Rev 40: 1153-1160, 2014.

20. Shetzer Y, Solomon H, Koifman G, Molchadsky A, Horesh S and Rotter V: The paradigm of mutant p53-expressing cancer stem cells and drug resistance. Carcinogenesis 35: 1196-1208, 2014.

21. Kubota E, Williamson CT, Ye R, Elegbede A, Peterson L, Lees-Miller SP and Bebb DG: Low ATM protein expression and depletion of p53 correlates with olaparib sensitivity in gastric cancer cell lines. Cell Cycle 13: 2129-2137, 2014.

22. Song MS, Salmena L and Pandolfi PP: The functions and regulation of the PTEN tumour suppressor. Nat Rev Mol Cell Biol 13: 283-296, 2012

23. Hollander MC, Blumenthal GM and Dennis PA: PTEN loss in the continuum of common cancers, rare syndromes and mouse models. Nat Rev Cancer 11: 289-301, 2011.

24. Carnero A, Blanco-Aparicio C, Renner O, Link W and Leal JFM: The PTEN/PI3K/AKT signalling pathway in cancer, therapeutic implications. Curr Cancer Drug Targets 8: 187-198, 2008.

25. Bjerre C, Vinther L, Belling KC, Würtz SØ, Yadav R, Lademann U, Rigina O, Do KN, Ditzel HJ, Lykkesfeldt AE, et al: TIMP1 overexpression mediates resistance of MCF-7 human breast cancer cells to fulvestrant and down-regulates progesterone receptor expression. Tumour Biol 34: 3839-3851, 2013.

26. Lu YC, Chang JT, Liao CT, Kang CJ, Huang SF, Chen IH, Huang CC, Huang YC, Chen WH, Tsai CY, et al: OncomiR-196 promotes an invasive phenotype in oral cancer through the NME4-JNK-TIMP1-MMP signaling pathway. Mol Cancer 13: 218,2014

27. Bunatova K, Pesta M, Kulda V, Topolcan O, Vrzalova J, Sutnar A, Treska V, Pecen L and Liska V: Plasma TIMP1 level is a prognostic factor in patients with liver metastases. Anticancer Res 32: 4601-4606, 2012. 